\title{
İztuzu Plajı (Dalyan-Muğla) ve Çevresinin Florası
}

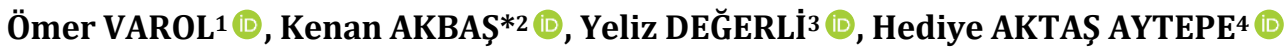 \\ 1,3,4Muğla Sıtkı Koçman Üniversitesi, Fen Fakültesi, Biyoloji Bölümü, 48000, Muğla, Türkiye \\ ${ }_{2}^{2}$ Muğla Sitkı Koçman Üniversitesi, Köyceğiz Meslek Yüksekokulu, Bitkisel ve Hayvansal Üretim Bölümü, 48800, \\ Muğla, Türkiye
}

(Alınış / Received: 28.03.2019, Kabul / Accepted: 03.09.2019, Online Yayınlanma / Published Online: 30.12.2019)

Anahtar Kelimeler
Dalyan,
Flora,
İztuzu,
Muğla

\section{Anahtar Kelimeler}

Muğla

\begin{abstract}
Özet: Bu çalışma, İztuzu plajının (Dalyan-Muğla) bitki çeşitliliğini belirlemek amacıyla gerçekleştirilmiștir. Araştırma alanı Davis'in kullandığı kareleme sistemine göre C2 karesinde bulunmaktadır. Çalışmamızda, Şubat 2016 - Kasım 2017 tarih aralığında çalışma alanından toplanan 264 bitki örneği değerlendirilmiş olup 54 familya, 77 cins, 240 tür, 1 alttür ve 1 varyete tespit edilmiştir. Endemik bitki taksonları 7 (\% 2.89) adettir. Bitki taksonlarının fitocoğrafik bölgelere göre dağılım oranları: Geniş yayılışlı ve yayılış alanları belli olmayan taksonların sayısı 113 (\%46.69). Akdeniz elementlerinin sayısı 75 (\%30.99), Doğu Akdeniz elementlerinin sayısı 47 (\%19.43), İran-Turan elementlerinin sayısı 2 (\%0.83) ve Avrupa-Sibirya elementlerinin sayısı 5 (\%2.06)'dir.
\end{abstract}

\section{Flora of İztuzu Beach (Dalyan-Muğla) and Its Surronding}

Keywords

Dalyan,

Flora,

İztuzu,

Muğla

\begin{abstract}
This study was conducted to determine the plant diversity of the İztuzu beach (Dalyan-Muğla). The research area is located in C2 square according to the grid system used by Davis. In our study, between February 2016 and November 2017, 264 plant samples collected from the study area were evaluated and 54 families, 77 genera, 240 species, 1 subspecies and 1 variety were determined. Endemic plant taxa are 7 (2.89\%). Distribution of plant taxa according to phytogeographical regions: The number of unknown in distribution areas and cosmopolits taxa are 113 (46.69\%). The number of Mediterranean elements are 75 (30.99\%), East Mediterranean elements are 47 (19.43\%), Irano-Turanian elements are $2(0.83 \%)$ and Euro-Siberian elements are 5 (2.06\%).
\end{abstract}

\section{Giriş}

Ülkemizin floristik zenginliğinin nedenleri arasında; jeolojik, jeomorfolojik çeşitlilik (yer şekilleri), iklim çeşitliliği, toprak çeşitliliği, bu çeşitliliklere bağlı olarak habitat çeşitliliği (ekolojik çeşitlilik) ve üç fitocoğrafik bölgenin (Avrupa-Sibirya, Akdeniz, İranTuran) varlığı saylabilir [1].

$\mathrm{Bu}$ nedenlerden dolayı Türkiye yerli ve yabancı araştırmacılar için önemli bir floristik merkez olarak değerlendirilmiștir. En güncel eser olan Türkiye Bitkileri Listesi (Damarlı Bitkiler) rakamlarına göre ülkemizdeki tür ve türaltı takson sayısı 11.707'dir ve bu sayı yeni bulunan bitki taksonları ile günden güne artış göstermektedir [2].

Ülkemizde floristik çalışmalar her ne kadar ileri seviyelerde olsa da, halen daha çalışılmamış bölgeler bulunmaktadır. Yapılan floristik çalışmalarla her geçen gün yeni türlerin keşfedilmesi, ülkemiz için yeni bitki türü kayıtlarının tespit edilmesi floristik çalışmaların ne kadar gerekli olduğunu gerçeğini ortaya koymaktadır.

\section{1. Çalışma alanının coğrafik özellikleri}

Köyceğiz-Dalyan Özel Çevre Koruma Bölgesi (ÖÇKB), Muğla iline bağlı Köyceğiz ve Ortaca ilçelerinin sınırları içerisinde yaklaşık $461 \mathrm{~km} 2$ genişliğinde bir alandır [3]. İztuzu plajı, Köyceğiz-Dalyan ÖÇKB içerisinde bulunan Dalyan beldesi yakınlarında 4,5 km uzunluğa sahip bir Akdeniz plajıdır (Şekil 1). Araștırma alanımız Akdeniz fitocoğrafik bölgesi içerisinde olup, Davis'in kullandığı kareleme sistemine göre C2 karesi içerisinde yer almaktadır [4]. 


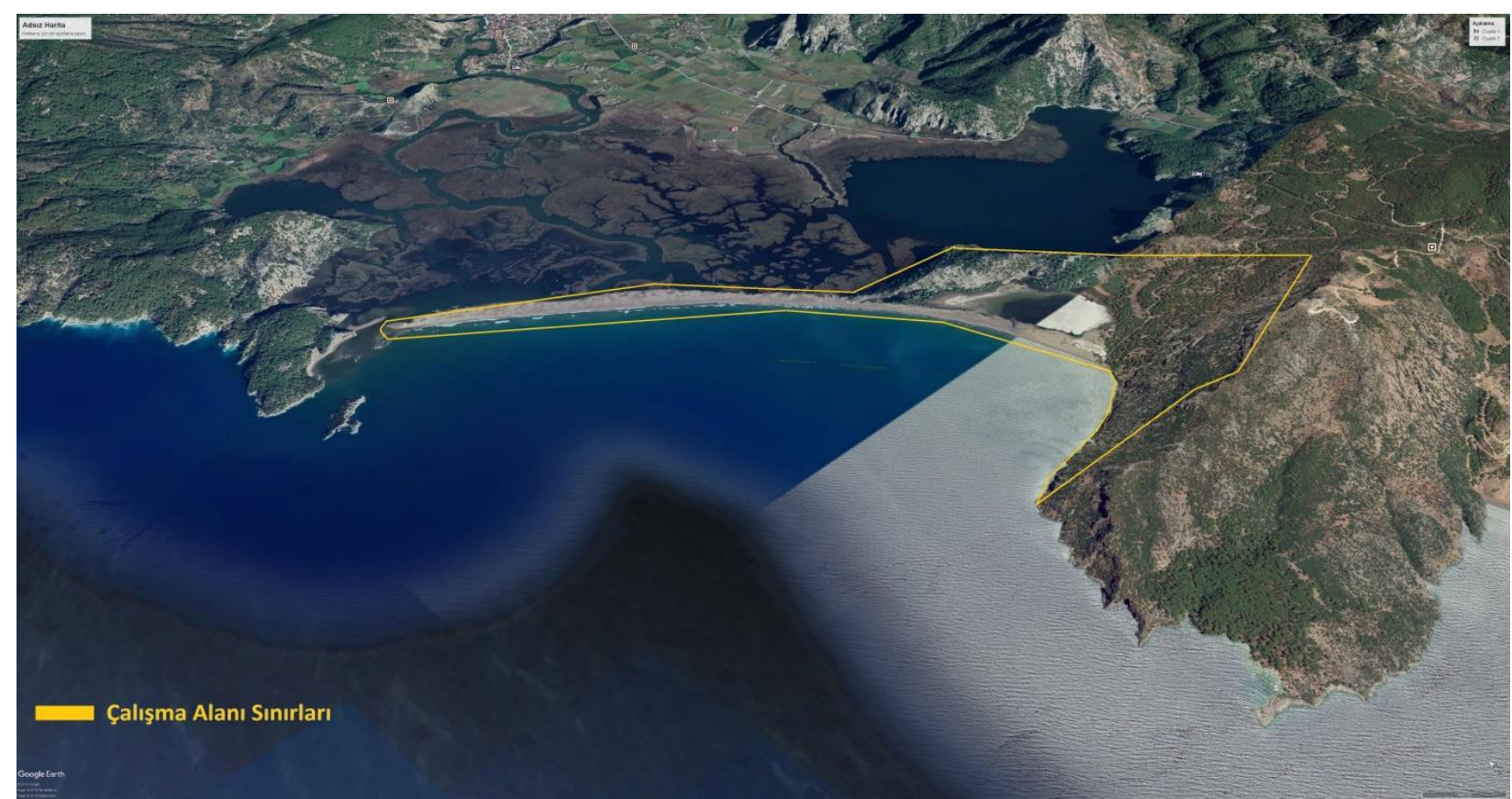

Şekil 1. Çalışma alanı haritası

\subsection{Araștırma alanının jeolojik yapısı ve büyük toprak grupları}

Araştırma alanın jeolojik yapısına bakıldığında, Kumsal çökeller ve Tavas Napı yer almaktadır [5]. Kumsal Çökelleri; sarımsı gri ve bej renkli, iyi boylanmış, yıkanmış kumlardan oluşmaktadır. Dik kıyı morfolojili alanlarda daha kaba taneli ve çakıllı olan bu kumlar özellikle Dalyan kanalların denize açıldığı bölge ile Bozburun Tepesi'nin kuzey eteklerinin denizle buluştuğu alan (İztuzu) arasında yüzeylenmektedir (Şekil 2).

Araştırma alanının büyük toprak grupları "Muğla İli
Arazi Varlığı" isimli kaynaktan faydalanılarak ortaya konmuştur. Çalışma alanımızda Kırmızı Kahverengi Akdeniz Toprakları ve Hidromorfik Alüvyal topraklar bulunmaktadır [6].

\subsection{Araştırma alanının iklimsel özellikleri}

Araştırma alanının iklim bilgileri, Muğla Meteoroloji Müdürlüğü'nün Köyceğiz ve Muğla (Merkez)'ya ait raporlarından alınmıştır. Elde edilen verilerle Köyceğiz ve Muğla'nın iklim diyagramları çizilmiştir (Şekil 3 ve 4). Köyceğiz istasyonunun yıllık sıcaklık ortalaması $18.3{ }^{\circ} \mathrm{C}$ 'dır. Muğla istasyonunun yıllık sıcaklık ortalaması $15.0{ }^{\circ} \mathrm{C}$ 'dir.

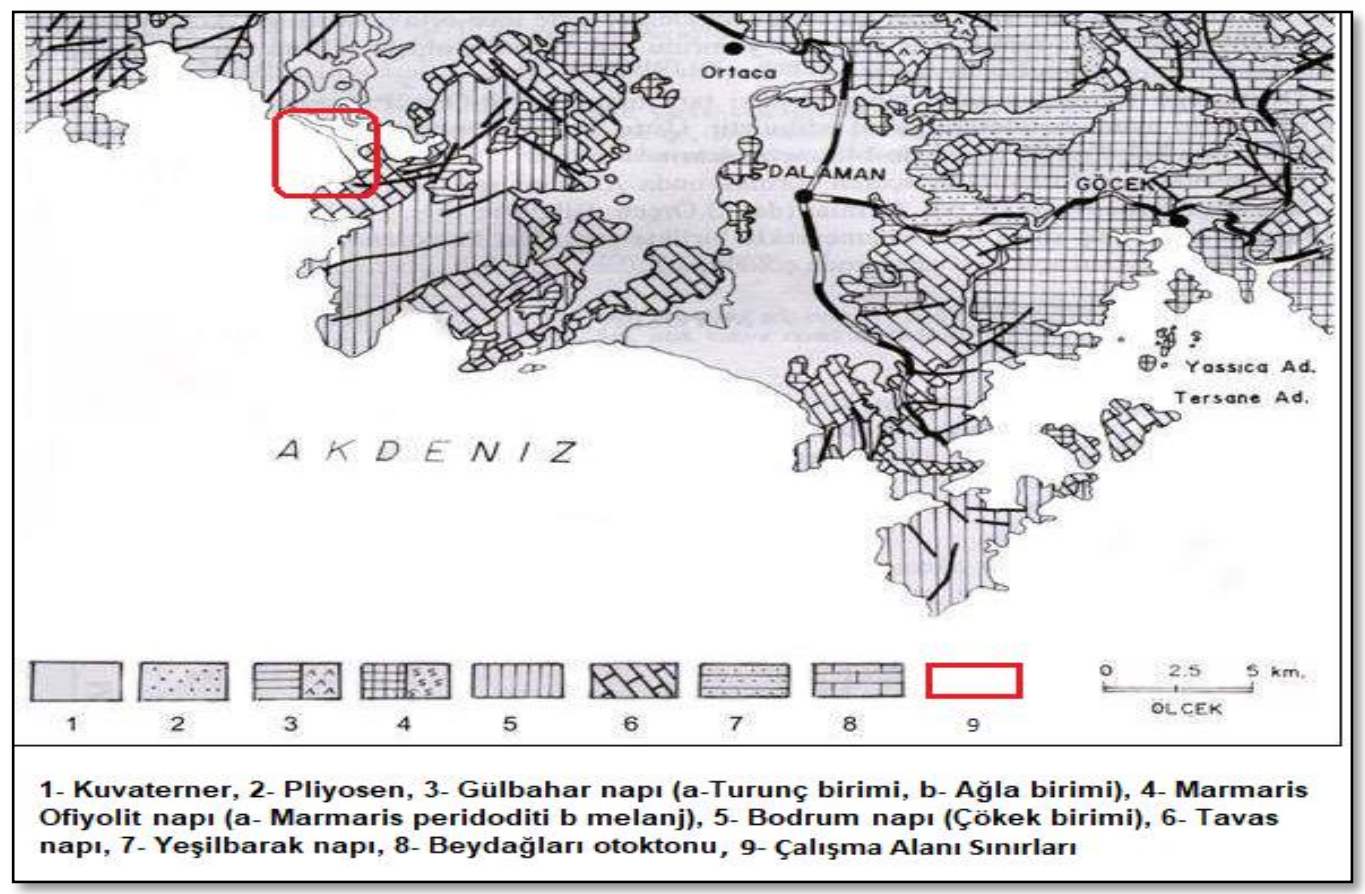

Şekil 2. Araştırma alanının jeoloji haritası [5] 


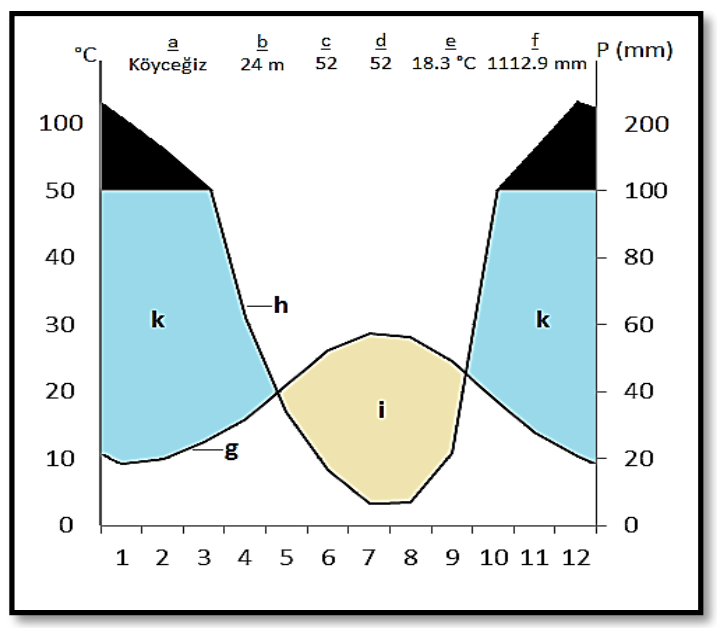

Şekil 3. Köyceğiz istasyonu ombrotermik diyagramı

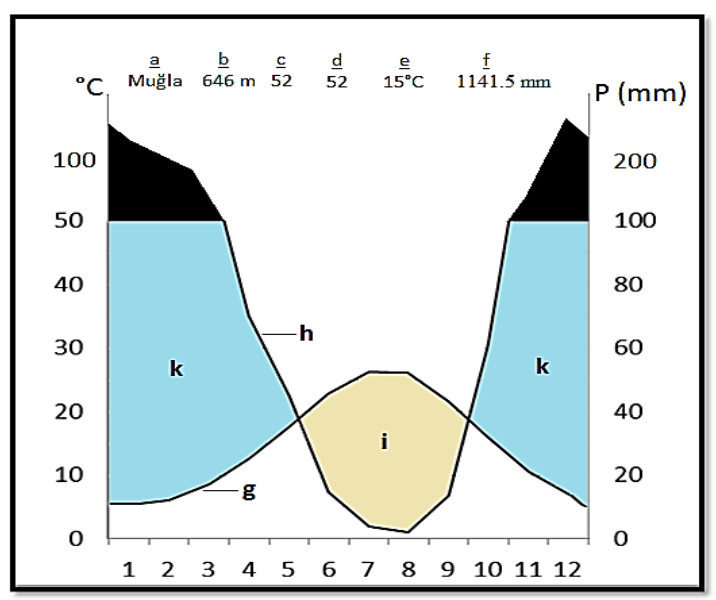

Şekil 4. Muğla istasyonu ombrotermik diyagramı

a. Meteoroloji istasyonunun ad, b. Meteoroloji istasyonunun rakımı (m), c. Sıcaklık rasat süresi, d. Yağış rasat süresi, e. Yıllık ortalama sıcaklık, f. Yıllık toplam yağış miktarı (mm), g. Sıcaklık eğrisi, h. Yağış eğrisi, i. Kurak periyot, k. Yağışlı periyot

Köyceğiz istasyonunda yıllık yağış ortalaması 73.5 mm'dir. Yağış rejimi KISY, Doğu Akdeniz Yağış Rejiminin 1. tipidir. Muğla istasyonunda yıllık yağış ortalaması 95.2 mm'dir. Yağış rejimi KISY, Doğu Akdeniz Yağış Rejiminin 1. tipidir.

Alanın iklim tipini belirlemek için Emberger'in yağış sıcaklık emsali (Q=2000.P/M2-m2) formülü kullanılmıştır [7-8].
Veriler santigrad derece $\left({ }^{\circ} \mathrm{C}\right)$ olarak kullanılmak istendiği için formül $\mathrm{Q}=2000 . \mathrm{P} /(\mathrm{M}+\mathrm{m}+546.4) \cdot(\mathrm{M}-\mathrm{m})$ şekline çevrilmiştir.

Q>98 ve $P>1000 \mathrm{~mm}$ yağış ise Yağışlı Akdeniz Biyoiklim Katı

m değerine göre Akdeniz biyoiklim katlarının alt tipleri;

$0^{\circ} \mathrm{C}<\mathrm{m}<3{ }^{\circ} \mathrm{C}$ ise Kışı Serin

$3^{\circ} \mathrm{C}<\mathrm{m}<4.5^{\circ} \mathrm{C}$ ise Kışı Ilıman

Alınan verilere göre Köyceğiz Yağışlı Ilık Akdeniz İklimi, Muğla Yağışlı Serin Akdeniz İklimine sahiptir (Tablo 1).

\section{Materyal ve Metot}

Araştırmamızın temel materyalini İztuzu Plajı ve çevresinden Şubat 2016 - Ağustos 2017 tarihleri arasında yapılmış olan arazi çalışmaları neticesinde toplanan 264 bitki örneği oluşturmaktadır. Araştırmamız, arazi ve laboratuvar çalışmaları olmak üzere iki aşamalı olarak gerçekleştirilmiştir.

\subsection{Arazi çalışması}

Araştırma bölgesine, 2016-2017 tarihleri arasında 18 ay boyunca gidilerek bitki örnekleri toplanmıștır. Toplanan bitki örnekleri, arazi defterine; numara verilerek, tarihi, morfolojik özellikleri, toplandığ yükselti, lokalite ve habitat özellikleri yazılarak kayıt altına alınmıştır.

\subsection{Laboratuvar çalışması}

Tüm bitki örnekleri preslenmiş ve herbaryum materyali haline getirilmiştir. Bitki numuneleri Davis'in "Flora of Turkey and The East Aegean Island" isimli 10 ciltlik eserinden ve bölgede yapılmış olan revizyon çalışmalarından faydalanılarak teşhis edilmiştir $[9,10,11]$. Bitki taksonlarının isimleri "Türkiye Bitkileri Listesi (Damarlı Bitkiler)" isimli kaynağa göre güncellenmiştir [2]. Teşhisi yapılarak, herbaryum materyali haline getirilen bitki numuneleri üniversitemiz herbaryumunda (MUH) saklanmaktadır.

Tablo 1. İstasyonların biyoiklim analizi tablosu

\begin{tabular}{|c|c|c|c|c|c|c|c|c|}
\hline İstasyon & $\begin{array}{c}\text { Yükseklik } \\
(\mathbf{m})\end{array}$ & $\begin{array}{c}\mathbf{P} \\
(\mathbf{m m})\end{array}$ & $\mathbf{M}$ & $\mathbf{m}$ & $\mathbf{Q}$ & $\mathbf{P E}$ & $\mathbf{P E} / \mathbf{M}$ & İklim Tipi \\
\hline Köyceğiz & 24 & 1112.9 & 36.2 & 4.0 & 117,84 & 27.1 & 0.75 & Yağışl, Ilık Akdeniz İklimi \\
\hline Muğla & 646 & 1141.5 & 33.3 & 1.5 & 123.64 & 20.3 & 0.61 & Yağışlı, Serin Akdeniz İklimi \\
\hline
\end{tabular}

$\mathrm{Q}=$ Yağış-sıcaklık emsali, $\mathrm{P}=$ Yıllık yağış miktarı $(\mathrm{mm})$, PE= Haziran-Temmuz-Ağustos ayları yağış toplamı, M= En sıcak ayın maksimum sıcaklık ortalaması $\left({ }^{\circ} \mathrm{C}\right), \mathrm{m}=$ En soğuk ayın minimum sıcaklık ortalaması $\left({ }^{\circ} \mathrm{C}\right)$ 


\section{Bulgular}

\subsection{Lokaliteler}

Çalışma alanımızdaki bitki numuneleri farklı lokalitelerden toplanmış olup, lokalitelerin listesi aşağıda verilmiştir;

Lok. 1. Muğla: Dalyan, İztuzu plajı, Kumul alanlar, 1-3 $\mathrm{m}$

Lok. 2. Muğla: Dalyan, İztuzu plajı doğu yamaçları, Maki açıklıkları, 20-50 m

Lok. 3. Muğla: Dalyan, İztuzu plajı güney yamaçları, Maki açıklıkları, 3-20 m

Lok. 4. Muğla: Dalyan, İztuzu plajı doğusu, Maki açıklıkları, kayalık yamaçlar, $50 \mathrm{~m}$

Lok. 5. Muğla: Dalyan, İztuzu plajı kuzey-batı yamaçlar, Makilik alanlar, 5-50 m

Lok. 6. Muğla: Dalyan, İztuzu plajı doğu yamaçları, Caretta caretta L. rehabilitasyon merkezi arkaları, Makilik alanlar, 3-10 m

Lok. 7. Muğla: Dalyan, İztuzu plajı doğu yamaçları, Pinus brutia orman altları, $10-100 \mathrm{~m}$

Lok. 8. Muğla: Dalyan, İztuzu plajı doğu yamaçları, Pinus brutia orman açıklığı, Sulak alanlar, $3 \mathrm{~m}$

Lok. 9. Muğla: Dalyan, İztuzu plajı doğu yamaçları, Caretta caretta L. rehabilitasyon merkezi arkaları, Pinus brutia orman açıklıkları, 2-10 m

Lok. 10. Muğla: Dalyan, İztuzu plajı doğu yamaçları, Caretta caretta L. rehabilitasyon merkezi arkalarl, Pinus brutia orman altları, 5-100 m

Lok.11. Muğla: Dalyan, İztuzu plajı batı ucu, Tur teknelerinin kalkış noktası çevresindeki kumul alanlar, 1-4 m

Lok. 12. Muğla: Dalyan, İztuzu plajı doğu yamaçları, Makilik alanlar, 2-5 m

Lok.13. Muğla: Dalyan, İztuzu plajı, İztuzu gölü arkasındaki makilik alanlar, 3-50 m

Lok.14. Muğla: Dalyan, İztuzu plajı, İztuzu gölü arkası Pinus brutia açıklıkları kayalık yamaçlar, 20-50 m

Lok.15. Muğla: Dalyan, İztuzu plajı, İztuzu gölü arkası Pinus brutia açıklıkları, 20-50 m

Lok.16. Muğla: Dalyan, İztuzu plajı, İztuzu gölü arkası Pinus brutia altları 10-50 m

\subsection{Floristik Liste}

\section{DIVISIO: PTERIDOPHYTA}

1. EQUISETACEAE

Equisetum palustre L., Lok. 11, 24.04.16, Ö.V. 7256

\section{DIVISIO: GYMNOSPERMAE}

\section{PINACEAE}

Pinus brutia Ten. var. brutia D. Akd. ele. Lok. 9, 27.02.16, Ö.V. 7040-a

\section{EPHEDRACEAE}

Ephedra foeminea Forssk. Lok. 12, 22.01.17, Ö.V. 7405

\section{DIVISIO: ANGIOSPERMAE}

\section{RANUNCULACEAE}

Anemone coronaria L. Akd. ele. Lok. 9, 27.02.16, Ö.V. 7045
Clematis cirrhosa L. Akd. ele. Lok. 12, 22.01.17, Ö.V. 7404

Ranunculus paludosus Poir. Lok. 9, 22.03.16, Ö.V. 7108, 7171

Ranunculus creticus L. Lok. 9, 27.02.16, Ö.V. 7036

Ranunculus muricatus L. Lok. 9, 09.04.16, Ö.V. 7172

Ranunculus ficaria L. subsp. ficariiformis Rouy \&Fouc. Lok. 9, 18.02.17, Ö.V. 7415

Staphisagria macrosperma Spach. Akd. ele. Lok. 10, 07.05.16, Ö.V. 7285

\section{PAPAVERACEAE}

Papaver gracile Boiss. Akd. ele. Lok. 2, 09.04.16, Ö.V. 7198

Papaver rhoeas L. Lok. 9, 09.04.16, Ö.V. 7193

\section{BRASSICACEAE}

Arabis verna (L.) DC. Akd. ele. Lok. 9, 27.02.16, Ö.V. 7050

Biscutella didyma L. Lok. 9, 27.02.16, Ö.V. 7042

Brassica nigra (L.) Koch Lok. 11, 24.04.16, Ö.V. 7258 Cakile maritima Scop. Lok. 1, 27.02.16, Ö.V. 7065

Capsella bursa-pastoris (L.) Medik. Lok. 9, 27.02.16, Ö.V. 7051

Cardamine graeca L. Lok. 2, 27.02.16, Ö.V. 7057

Cardamine hirsuta L. Lok. 10, 18.02.17, Ö.V. 7413

Clypeola janthlaspi L. Lok. 5, 22.03.16, Ö.V. 7119

Draba verna L. Lok. 10, 18.02.17, Ö.V. 7417

Microthlaspi perfoliatum (L.) F.K. Mey. Lok. 9, 27.02.16, Ö.V. 7041

Malcolmia flexuosa Sibth \& Sm. D. Akd. ele. Lok. 9, 27.02.16, Ö.V. 7040

Nasturtium officinale R. Br. Lok. 8, 09.04.16, Ö.V. 7184

Sisymbrium officinale (L.) Scop, Lok. 9, 09.04.16, Ö.V. 7170

\section{CARYOPHYLLACEAE}

Arenaria graveolens Schreb. D. Akd ele Lok. 13, 07.05.16, Ö.V. 7296

Arenaria pamphylica Boiss.\&Hohen. subsp. pamphylica var. pamphylica End. Akd. ele. Lok. 13, 29.05.16, Ö.V. 7318

Cerastium glomeratum Thuill. Lok. 9, 22.03.16, Ö.V. 7098

Minuartia hybrida (Vill.) Schischk. subsp. hybrida Lok. 9, 22.03.16, Ö.V. 7099

Polycarpon tetraphyllum L. Lok. 9, 07.05.16, Ö.V. 7287

Petrorhagia dubia (Raf.) G.Lopez \& Romo Lok. 9, 09.04.16, Ö.V. 7168

Stellaria neglecta (Weihe) Gremli Lok. 9, 27.02.16, Ö.V. 7048

Stellaria media (L.) Vill. Lok. 9, 27.02.16, Ö.V. 7035

Silene cretica L. Akd. ele. Lok. 9, 09.04.16, Ö.V. 7185

Silene gallica L. Lok. 1, 09.04.16, Ö.V. 7161

Velezia rigida L. Lok. 13, 07.05.16, Ö.V. 7294

\section{POLYGONACEAE}

Rumex tuberosus L. subsp. creticus (Boiss.) Rech

D. Akd. ele Lok. 9, 22.03.16, Ö.V. 7100 
Rumex bucephalophorus L. subsp. bucephalophorus Akd. ele. Lok. 9, 22.03.16, Ö.V. 7109

\section{AMARANTHACEAE}

Chenopodium murale L. Lok. 9, 29.05.16, Ö.V. 7316

Salsola kali L. Lok. 1, 29.05.16, Ö.V. 7311

\section{LINACEAE}

Linum bienne Miller, Lok. 1, 27.02.16, Ö.V. 7026

Linum strictum L. var. spicatum Pers. Lok. 11, 24.04.16, Ö.V. 7257

\section{MALVAECEAE}

Malva sylvestris L. Lok. 12, 24.04.16, Ö.V. 7282

Malva nicaeensis All. Lok. 12, 24.04.16, Ö.V. 7281

\section{GERANIACEAE}

Geranium purpureum Vill. Lok. 9, 22.03.16, Ö.V. 7067

Geranium lucidum L. Lok. 9, 27.02.16, Ö.V. 7044

Geranium molle L., Lok. 9, 27.02.16, Ö.V. 7033

Erodium cicutarium (L.) L'Hérit subsp. cicutarium, Lok. 9, 27.02.16, Ö.V. 7032

Erodium cicutarium (L.) L' Herit. subsp. bipinnatum (Desf.) Tourlet Lok. 1, 29.05.16, Ö.V. 7307

\section{ANACARDIACEAE}

Pistacia palaestina Boiss. D. Akd. ele. Lok. 9, 09.04.16, Ö.V. 7192

\section{FABACEAE}

Alhagi maurorum Medik. subsp. maurorum Ir. Tur. ele. Lok. 11, 13.08.16, Ö.V. 7342

Ceratonia siliqua L. Akd. ele. Lok. 12, 17.03.17, Ö.V. 7428

Hymenocarpus circinnatus (L.) Savi Lok. 9, 22.03.16, Ö.V. 7107

Lotus halophilus Boiss. \& Sprun. Lok. 1, 09.04.16, Ö.V. 7204

Medicago littoralis Rohde ex Lois var. littoralis, Lok. 1, 27.02.16, Ö.V. 7025, Lok. 5, 22.03.16, Ö.V. 7114 Medicago marina L., Lok. 1, 27.02.16, Ö.V. 7024

Medicago rigidula (L.) All. var. rigidula Lok. 1, 29.05.16, Ö.V. 7304

Melilotus indicus (L.) All. Lok. 1, 24.04.16, Ö.V. 7268 Onobrychis caput-galli (L.) Lam. Akd. ele. Lok. 1, 09.04.16, Ö.V. 7148

Securigera parviflora (Desv.) Lassen D. Ekd. ele. Lok. 9, 22.03.16, Ö.V. 7095

Securigera cretica (L.) Lassen Lok. 1, 09.04.17, Ö.V. 7460

Scorpiurus subvillosus L. var. subvillosus (L.) Fiori Lok. 3, 09.04.16, Ö.V. 7202

Tripodion tetraphyllum (L.) Fourr. Akd. ele. Lok. 1, 09.04.16, Ö.V. 7156

Trifolium clypeatum L. Lok. 7, 22.03.16, Ö.V. 7112

Trifolium campestre Schreb. subsp. campestre var. campestre Lok. 1, 09.04.16, Ö.V. 7142
Trifolium hirtum All. Akd. ele. Lok. 1, 09.04.17, Ö.V. 7459

Trifolium lucanicum Gasp. Akd. ele. Lok. 1, 09.04.16, Ö.V. 7150

Trifolium Iappaceum L. Akd. ele. Lok. 1, 09.04.16, Ö.V. 7154

Trifolium tomentosum L. var. tomentosum Lok. 1, 09.04.16, Ö.V. 7155

Trifolium stellatum L. var. stellatum Lok. 9, 09.04.16, Ö.V. 7175

Trifolium grandiflorum Schreb. D. Akd. ele. Lok. 9, 09.04.16, Ö.V. 7173

Trifolium nigrescens Viv. subsp. petrisavii (Clem.) Holmboe Lok. 2, 24.04.16, Ö.V. 7274

Trigonella spruneriana Boiss. var. spruneriana Ir.Tur. ele. Lok. 1, 09.04.16, Ö.V. 7157

Trigonella monspeliaca L. Lok. 1, 09.04.17, Ö.V. 7456

Vicia hybrida L. Lok. 9, 22.03.16, Ö.V. 7101

\section{ROSACEAE}

Pyrus amygdaliformis Vill. var. amygdaliformis D. Akd. ele. Lok. 2, 24.04.16, Ö.V. 7273

\section{MYRTACEAE}

Eucalyptus camaldulensis Dehnh. subsp. camaldulensis (Kültür)

\section{CRASSULACEAE}

Rosularia serrata (L.) Berger D. Akd. ele. Lok. 14, 07.05.16, Ö.V. 7295

\section{APIACEAE}

Bupleurum gracile d'Urv. Akd. ele. Lok. 3, 24.04.16, Ö.V. 7262

Daucus broteri Ten. Akd. ele. Lok. 1, 09.04.16, Ö.V. 7205

Daucus guttatus Sm. Lok. 9, 09.04.16, Ö.V. 7191

Eryngium maritimum L. Lok. 1, 29.05.16, Ö.V. 7310

Lagoecia cuminioides L. Akd. ele. Lok. 3, 24.04.16, Ö.V. 7263

Orlaya daucoides (L.) Greuter Akd. ele. Lok. 9, 22.03.16, Ö.V. 7097

Pseudorlaya pumila (L.) Grande Akd. ele. Lok. 1, 29.05.16, Ö.V. 7306

Scandix pecten-veneris L. Lok. 9, 22.03.16, Ö.V. 7094 Smyrnium rotundifolium Mill. D. Akd. ele. Lok. 5, 20.05.17, Ö.V. 7526

Torilis arvensis (Huds.) Link subsp. purpurea (Ten.) Hayek Akd. ele. Lok. 10, 09.04.16, Ö.V. 7181 Torilis nodosa (L.) Gaertner Lok. 10, 09.04.16, Ö.V. 7182

Torilis leptophylla (L.) Reichb. Lok. 10, 09.04.16, Ö.V. 7183

\section{CAPRIFOLIACEAE}

Knautia integrifolia (L.) Bert var. bidens (Sm.) Borbàs D. Akd. ele. Lok. 3, 09.04.16, Ö.V. 7200 Valeriana dioscoridis Sm. D. Akd. ele. Lok. 2, 27.02.16, Ö.V. 7059 
Valerianella obtusiloba Boiss. D. Akd. ele. Lok. 9, 22.03.16, Ö.V. 7096

\section{ASTERACEAE}

Anthemis chia L. D. Akd. ele. Lok. 9, 27.02.16, Ö.V. 7041-a

Anthemis cretica L. subsp. anatolica (Boiss.) Grierson, Lok. 9, 27.02.16, Ö.V. 7034

Anthemis pseudocotula Boiss. Lok. 1, 09.04.16, Ö.V. 7211

Asteriscus spinosus (L.) Sch. Bip. Akd. ele. Lok. 5, 29.05.16, Ö.V. 7314

Bellis perennis L., Avr.-Sib. ele. Lok. 9, 27.02.16, Ö.V. 7030

Carthamus lanatus L. Lok. 5, 29.05.16, Ö.V. 7315

Crepis sancta (L.) Babcock, Lok. 9, 27.02.16, Ö.V. 7031

Conyza bonariensis (L.) Cronquist Lok. 11, 13.08.16, Ö.V. 7346

Cirsium creticum (Lam.) d' Urv. subsp. creticum Akd. ele. Lok. 9, 09.04.16, Ö.V. 7165

Cichorium pumilum Jacq. D. Akd. ele. Lok. 10, 07.05.16, Ö.V. 7186

Filago eriocephala Guss. D. Akd. ele. Lok. 9, 27.02.16, Ö.V. 7042-a

Glebionis segetum (L.) Fourr. Lok. 10, 24.04.16, Ö.V. 7277

Hedypnois rhagadioloides (L.) F.W. Schmidt subsp. cretica (L.) Hayek Akd. ele. Lok. 1, 09.04.17, Ö.V. 7462

Hyoseris scabra L. Akd. ele. Lok. 9, 22.03.16, Ö.V. 7104

Helichrysum stoechas (L.) Moench subsp. barrelieri (Ten.) Nyman Lok. 1, 09.04.17, Ö.V. 7453

Inula heterolepis Boiss. D. Akd. ele. Lok. 14, 07.05.16, Ö.V. 7292

Inula graveolens (L.) Desf. Akd. ele. Lok. 1, 03.11.16, Ö.V. 7395

Leontodon tuberosus L. Akd. ele. Lok. 7, 27.02.16, Ö.V. 7064

Phagnalon graecum Boiss. D. Akd. ele. Lok. 3, 09.04.16, Ö.V. 7201

Ptilostemon chamaepeuce (L.) Less. D. Akd. ele. Lok. 4, 09.04.16, Ö.V. 7194

Picris hieracioides L. subsp. hieracioides Avr.-sib. ele. Lok. 3, 24.04.16, Ö.V. 7267

Picnomon acarna (L.) Cass. Lok. 9, 10.07.16, Ö.V. 7340

Rhagadiolus stellatus (L.) Gaertner. Akd. ele. Lok. 9, 22.03.16, Ö.V. 7102

Senecio vulgaris L., Lok. 9, 27.02.16, Ö.V. 7027

Senecio vernalis Waldst. \& Kit. Lok. 10, 18.02.17, Ö.V. 7420

Tragopogon porrifolius L. subsp. longirostris (Sch. Bip.) Greuter Lok. 3, 24.04.16, Ö.V. 7265

Taraxacum hellenicum Dahlst. Akd. ele. Lok. 7, 03.11.16, Ö.V. 7400

Urospermum picroides (L.) F.W. Schmidt Akd. ele. Lok. 9, 22.03.16, Ö.V. 7105

18. CAMPANULACEAE
Campanula drabifolia SM. Lok. 5, 22.03.16, Ö.V. 7118, Lok. 9, 09.04.16, Ö.V. 7167

Campanula hagielia Boiss. Lok. 4, 09.04.16, Ö.V. 7195

\section{PRIMULACEAE}

Cyclamen alpinum Dammann ex. Sprenger End. D. Akd. ele. Lok. 2, 27.02.16, Ö.V. 7061

Anagallis arvensis L. var. caerulea (L.) Gouan Lok. 9, 22.03.16, Ö.V. 7106

Anagallis arvensis L. var. arvensis Lok. 9, 09.04.16, Ö.V. 7189

\section{APOCYNACEAE}

Nerium oleander L. Akd. ele. Lok. 1, 29.05.16, Ö.V. 73012

\section{OLEACEAE}

Olea europaea L. var. europaea Akd.ele Lok. 2, 27.02.16, Ö.V. 7059-a

\section{GENTIANACEAE}

Blackstonia perfoliata (L.) Hudson subsp. perfoliata Lok. 1, 24.04.16, Ö.V. 7270

Centarium pulchellum (Swartz) Druce Lok. 1, 09.04.16, Ö.V. 7143

Centaurium erythraea Rafn subsp. rhodense Akd. ele. Lok. 9, 29.05.16, Ö.V. 7317

\section{CONVOLVULACEAE}

Ipomoea imperati (Vahl) Griseb Akd. ele. Lok. 11, 13.08.16, Ö.V. 7345

\section{BORAGINACEAE}

Onosma frutescens Lam. D. Akd. ele. Lok. 5, 27.02.16, Ö.V. 7067

Alkanna mughlae Güner, H. Duman \& Şağban End. D. Akd. ele. Lok. 4, 09.04.16, Ö.V. 7196, 24.04.16, Ö.V. 7268

Echium plantagineum L. Akd. ele. Lok. 10, 24.04.16, Ö.V. 7278

Myosotis ramosissima Rochel. Oesterr. Fl. Lok. 9, 09.04.16, Ö.V. 7174

\section{SOLANACEAE}

Solanum americanum Mill. Lok. 9, 09.04.16, Ö.V. 7166

\section{SCROPHULARIACEAE}

Verbascum symes Murb. \& Rech. Fil. D. Akd. ele. Lok. 15, 07.05.16, Ö.V. 7290

Scrophularia pinardii Boiss. D. Akd. ele. Lok. 2, 27.02.16, Ö.V. 7058

Scrophularia peregrina L. Akd. ele. Lok. 9, 09.04.16, Ö.V. 7187

\section{LAMIACEAE}

Ballota acetabulosa (L.) Bentham D. Akd. ele. Lok. 3, 24.04.16, Ö.V. 7261 
Clinopodium nepeta (L.) Kuntze subsp. glandulosum (Req.) Govaerts Avr.-Sib. ele. Lok. 9, 10.07.16, Ö.V. 7341

Lamium amplexicaule L. var. amplexicaule Lok. 9, 27.02.16, Ö.V. 7049

Lamium moschatum Miller var. moscahtum Akd. ele. Lok. 9, 27.02.16, Ö.V. 7037

Micromeria myrtifolia Boiss.\&Hohen. Lok. 5, 29.05.16, Ö.V. 7313

Origanum onites L. Lok. 15, 07.05.16, Ö.V. 7291

Phlomis lycia D. Don. D. Akd. ele. Lok. 5, 22.03.16, Ö.V. 7116

Salvia viridis L. Akd. ele. Lok. 5, 22.03.16, Ö.V. 7113

Sideritis romana L. subsp. curvidens (Stapf)

Holmboe D. Akd. ele. Lok. 5, 22.03.16, Ö.V. 7115

Teucrium divaricatum Sieber subsp. divaricatum Akd. ele. Lok. 3, 24.04.16, Ö.V. 7266

Teucrium polium L. subsp. polium Lok. 13, 07.05.16, Ö.V. 7295-a

Vitex agnus-castus L. Akd. ele. Lok. 1, 10.07.16, Ö.V. 7339

\section{OROBANCHACEAE}

Orobanche nana Noe ex G. Beck Lok. 1, 09.04.16, Ö.V. 7163

Orobanche minor SM. Lok. 9, 09.04.16, Ö.V. 7169

Parentucellia latifolia (L.) Caruel subsp. Iatifolia Akd. ele. Lok. 1, 09.04.16, Ö.V. 7147

\section{PLUMBAGINACEAE}

Limonium virgatum (Wild.) Fourr. Akd. ele. Lok. 1, 10.07.16, Ö.V. 7337

\section{PLANTAGINACEAE}

Plantago lagopus L., Akd. ele., Lok. 9, 27.02.16, Ö.V. 7029

Plantago scabra Moench Lok. 1, 24.04.16, Ö.V. 7284

Plantago cretica L. D. Akd. ele. Lok. 1, 09.04.16, Ö.V. 7160

Plantago coronopus L. subsp. commutata (Guss.) Pilger D. Akd. ele. Lok. 1, 09.04.16, Ö.V. 7162

Kickxia commutata (Bernh. Ex Reichb.) Fritsch subsp. commutata Akd. ele. Lok. 13, 07.05.16, Ö.V. 7297

Veronica stamatiadae M.A. Fischer \& Greuter Akd. ele. Lok. 7, 22.03.16, Ö.V. 7110

Veronica anagallis-aquatica L. Lok. 8, 24.04.16, Ö.V. 7283

Veronica cymbalaria Bodard Akd. ele. Lok. 9, 27.02.16, Ö.V. 7039

\section{THYMELACEAE}

Daphne gnidioides Jaub \& Spach D. Akd. ele. Lok. 9, 27.02.16, Ö.V. 7047

Thymelaea hirsuta (L.) Endl. Akd. ele. Lok. 1, 27.02.16, Ö.V. 7066

\section{LAURACEAE}

Laurus nobilis L. Akd. ele. Lok. 9, 27.02.16, Ö.V. 7046

\section{ARISTOLOCHIACEAE}

Aristolochia guichardii Davis \& Khan End. D. Akd. ele. Lok. 10, 09.04.16, Ö.V. 7180

\section{EUPHORBIACEAE}

Mercurialis annua L. Lok. 9, 27.02.16, Ö.V. 7056

Euphorbia acanthothamnos Heldr. \& Sart ex Boiss D. Akd. ele. Lok. 5, 22.03.16, Ö.V. 7120

Euphorbia peplus L. var. peplus, Lok. 9, 27.02.16, Ö.V. 7028

Euphorbia characias L. subp. wulfenii (Hoppe ex W. Koch) A.R. Smith D. Akd. ele. Lok. 2, 27.02.16, Ö.V. 7060

Euphorbia paralias L. Akd. ele. Lok. 1, 09.04.16, Ö.V. 7199

Euphorbia helioscopia L. subsp. helioscopia Lok. 12, 24.04.16, Ö.V. 7279

Euphorbia peplis L. Akd. ele. Lok. 1, 29.05.16, Ö.V. 7308

\section{URTICACEAE}

Parietaria lusitanica L. Akd. ele. Lok. 7, 27.02.16, Ö.V. 7062

Urtica membranacea Poiret Akd. ele. Lok. 9, 27.02.16, Ö.V. 7038

Urtica pilulifera L. Akd. ele. Lok. 11, 24.04.16, Ö.V. 7251

\section{FAGACEAE}

Quercus aucheri Jaub. \& Spach End. D. Akd. ele. Lok. 5, 22.03.16, Ö.V. 7117

\section{MORACEAE}

Ficus carica L. subsp. carica Akd. ele. Lok. 11, 13.08.16, Ö.V. 734

\section{ULMACEAE}

Ulmus canescens Merville D. Akd. ele. Lok. 11, 13.08.16, Ö.V. 7343

\section{RUBIACEAE}

Galium aparine L. Lok. 9, 22.03.16, Ö.V. 7103

Galium brevifolium Sm. subsp. brevifolium End. D.Akd. ele. Lok. 9, 27.02.16, Ö.V. 7055

Galium graecum L. subsp. graecum D. Akd. ele. Lok. 14, 07.05.16, Ö.V. 7293

Valantia hispida L. Akd. ele. Lok. 5, 27.02.16, Ö.V. 7069

Sherardia arvensis L. Akd. ele. Lok. 5, 27.02.16, Ö.V. 7068

\section{ARACEAE}

Arisarum vulgare Targ.-Tozz subsp. vulgare Akd. ele. Lok. 9, 27.02.16, Ö.V. 7052

\section{ORCHIDACEAE}

Ophrys candica (E. Nelson ex Soo) H. Baumann \& Künkele subsp. candica Akd. ele. Lok. 10, 09.04.16, Ö.V. 7176

Serapias bergonii E.G.Camus Akd. ele. Lok. 1, 09.04.16, Ö.V. 7151 
Orchis sancta L. D. Akd. ele. Lok. 12, 24.04.16, Ö.V. 7280

Barlia robertiana (Loisel.) Greuter Akd. ele. Lok. 10, 18.02.17, Ö.V. 7414

Spiranthes spiralis (L.) Chevall. Akd. ele. Lok. 9, 03.11.16, Ö.V. 7397

Cephalanthera epipactoides Fisch. \& Mey. D. Akd. ele. Lok. 7, 09.04.17, Ö.V. 7561

\section{LILIACEAE}

Gagea graeca (L.) Terracc. D. Akd. ele. Lok. 9, 22.03.16, Ö.V. 7093

Gagea peduncularis (C \& J Presl) Pascher Akd. Ele. Lok. 9, 27.02.16, Ö.V. 7043

Fritillaria sibthorpiana (Sm.) Baker subsp. enginiana Byfi eld \& N. Özhatay End. D. Akd. ele. Lok. 7, 27.02.16, Ö.V. 7063

\section{XANTHORRHOEACEAE}

Asphodelus aestivus Brot. Lok. 9, 27.02.16, Ö.V. 7054

\section{SMILACACEAE}

Smilax excelsa L. Avr-Sib. ele. Lok. 2, 27.02.16, Ö.V. 7059

\section{ASPARAGACEAE}

Drimia maritima (L.) Stearn. Lok. 7, 24.09.16, Ö.V. 7391

Muscari comosum (L.) Mill. Akd. ele. Lok. 9, 09.04.16, Ö.V. 7190

Ornithogalum narbonense L. Akd. ele. Lok. 2, 24.04.16, Ö.V. 7271

Prospero autumnale (L.) Speta Akd. ele. Lok. 9, 03.11.16, Ö.V. 7398

\section{AMARYLLIDACEAE}

Allium subhirsutum L. Akd. ele. Lok. 10, 09.04.16, Ö.V. 7177

Allium sphaerocephalon L. subsp. arvense (Guss.) Arc. D. Akd. ele. Lok. 5, 10.07.16, Ö.V. 7338

Allium ampeloprasum L. Akd. ele. Lok. 5, 20.05.17, Ö.V. 7525

Pancratium maritimum L. Akd. ele. Lok. 11, 24.09.16, Ö.V. 7391-a

\section{COLCHICACEAE}

Colchicum balansae Planchon D. Akd. ele. Lok. 7, 24.09.16, Ö.V. 7392

\section{IRIDACEAE}

Gladiolus anatolicus (Boiss.) Stapf D. Akd. ele Lok. 3, 09.04.16, Ö.V. 7203

Romulea tempskyana Freyn D. Akd. ele. Lok. 9, 18.02.17, Ö.V. 7416

\section{JUNCACEAE}

Juncus acutus L. subsp. acutus Lok. 1, 22.01.17, Ö.V. 7407

\section{CYPERACEAE}

Schoenus nigricans L. Lok. 1, 09.04.16, Ö.V. 7207 Carex divulsa Stokes subsp. divulsa Lok. 8, 09.04.16, Ö.V. 7188

Carex divisa Hudson, Lok. 11, 24.04.16, Ö.V. 7253

Cyperus capitatus Vandelli Lok. 11, 24.04.16, Ö.V. 7255

Scirpoides holoschoenus (L.) Sojak subsp. holoschoenus Lok. 11, 24.04.16, Ö.V. 7254

\section{POACEAE}

Aeluropus littoralis (Gouan) Park. Lok. 11, 13.08.16, Ö.V. 7347

Aegilops umbellulata Zhukovsky Lok. 3, 24.04.16, Ö.V. 7260

Andropogon distachyos L. Akd. ele. Lok. 1, 09.04.16, Ö.V. 7208

Avena barbata Pott ex Link subsp. barbata Akd. ele. Lok. 1, 09.04.16, Ö.V. 7153

Briza maxima L. Lok. 10, 09.04.16, Ö.V. 7179

Briza minor L. Lok. 10, 24.04.16, Ö.V. 7276

Bromus diandrus Roth Lok. 7, 22.03.16, Ö.V. 7111

Bromus fasciculatus C. Presl D. Akd. ele. Lok. 1, 09.04.16, Ö.V. 7145

Bromus intermedius Guss. Lok. 1, 09.04.16, Ö.V. 7209

Bromus hordeaceus L. subsp. hordeaceus Lok. 9, 09.04.16, Ö.V. 7186

Cynodon dactylon (L.) Pers. var. dactylon Lok. 1, 24.04.16, Ö.V. 7269

Cynosurus echinatus L. Akd. ele. Lok. 10, 09.04.16, Ö.V. 7178

Dactylis glomerata L. subsp. hispanica (Roth) Nyman Lok. 3, 24.04.16, Ö.V. 7264

Gastridium phleoides (Nees \& Meyen) C.E.Hubbard Akd. Ele. Lok. 7, 29.05.16, Ö.V. 7319

Hordeum murinum L. subsp. glaucum (Steudel) Tzvelev Lok. 1, 09.04.16, Ö.V. 7164

Imperata cylindrica (L.) Raeuschel Lok. 1, 09.04.16, Ö.V. 7206

Lagurus ovatus L. Akd. ele. Lok. 1, 09.04.16, Ö.V. 7146

Lolium rigidum Gaudin var. rigidum Lok. 1, 09.04.16, Ö.V. 7210

Lolium multiflorum Lam. Lok. 11, 24.04.16, Ö.V. 7250

Melica minuta L. Akd. ele. Lok. 2, 09.04.16, Ö.V. 7197

Poa bulbosa L. Lok. 1, 09.04.17, Ö.V. 7458

Phragmites australis (Cav.) Trin. Avr-Sib. ele. Lok. 1, 03.11.16, Ö.V. 7396

Polypogon maritimus Willd. subsp. subspathaceus (Req.) Aschers. \& Graebn. D. Akd. ele. Lok. 1, 09.04.16, Ö.V. 7157

Oryzopsis miliacea (L.) Asch. \& Schweinf. subsp. thomasii (Duby) K.Richt. Lok. 11, 24.04.16, Ö.V. 7252

Brachypodium distachyon (L.) P.Beauv. Akd. ele. Lok. 1, 09.04.16, Ö.V. 7149

Vulpia ciliata Dumort subsp. ciliata Lok. 1, 09.04.16, Ö.V. 7159

Vulpia fasciculata (Forsskal) Fritsch Akd. ele. Lok. 11, 24.04.16, Ö.V. 7259 


\section{Tartışma ve Sonuç}

Araştırma alanından 2016 Şubat ayından 2017 Ağustos ayına kadar geçen 18 aylık süre boyunca 264 bitki numunesi toplanmış ve bu numunelerin tayin edilmesiyle 54 familyaya ait 240 tür, 1 alttür ve 1 varyete olmak üzere toplamda 242 tür ve türaltı bitki taksonu belirlenmiștir. Belirlenen taksonlardan 7 adeti endemiktir. Bitki taksonlarının 1'i Pteridophyta, 2'si Gymnospermae, 239'u Angiospermae olarak belirlenmiştir.

Tespit edilen taksonların \%30.99 (75)'unu Akdeniz elementi, \%19.43 (47)'ünü Doğu Akdeniz elementi, \%2.06 (5)'sinı Avrupa-Sibirya elementi, \%0.83 (2)'ünü İran-Turan elementi ve \%46.69 (113)'luk kısmını da geniş yayılışlı veya fitocoğrafik bölgesi bilinmeyen taksonlar oluşturmaktadır (Şekil 5).

Çalışma alanımızın endemizm oranı \% 2.89'dur. Ülkemiz genelinde bu oran \% 30'un üzerinde olup, çoğu nadir ve endemik bitki taksonu yükseltisi fazla olan habitatlarda yayılış göstermektedir. Araştırma alanımız ile yakın bölgelerde gerçekleştirilmiş floristik çalışmaların endemizm oranına ait karşılaştırma Tablo 2'de verilmiştir [12-15]. Bu tablo incelendiğinde endemizm oranı karşılaştırılan tüm alanlara göre en düşük olan çalışmanın İztuzu Plajı ve Çevresinin Florası olduğu görülmektedir. Endemizm oranının düşük olmasının sebebinin çalışma bölgemizin deniz seviyesinde olmasından kaynaklandığı düşünülmektedir.

Endemizm oranı en yüksek olan çalışmanın KöyceğizDalyan Özel Koruma Alanının Florasında olduğu görülmektedir. $\mathrm{Bu}$ durumun nedeninin ise Özel Koruma Alanının çalışma alanımıza kıyasla çok daha büyük bir alanı ve farklı vejetasyon tiplerini barındırmasından kaynaklandığı düşünülmektedir.

Urban floranın çalışıldığı Muğla şehir merkezi ile Labranda florasının çalışıldığı bölge hemen hemen aynı yükselti aralığında bulunmakta ve bu durumun endemizm oranındaki yakınlığı açıkladığı kanaatindeyiz.

Aspat kalesi ve çevresinin çalışıldığı floristik araştırmada endemizm oranının bu kadar düşük olmasının; çalışma alanının oldukça küçük bir yüz ölçüme sahip olması, fazla vejetasyon serilerini barındırmaması ve en önemlisi rakımın 280 m'yi geçmeyişinden kaynaklandığı kanaatindeyiz.

Çalıșma alanımızdan toplanan endemik bitkilerin tehlike kategorileri IUCN kriterleri, Türkiye bitkileri kırmızı kitabından bakılarak kategorize edilmiştir $[16,17]$. Sonuçlara göre iki tür tehlike (EN) kategorisinde, iki tür zarar görebilir (VU) kategorisinde ve üç tür en az endişe verici (LC) kategorisinde yer almaktadır (Tablo 3).

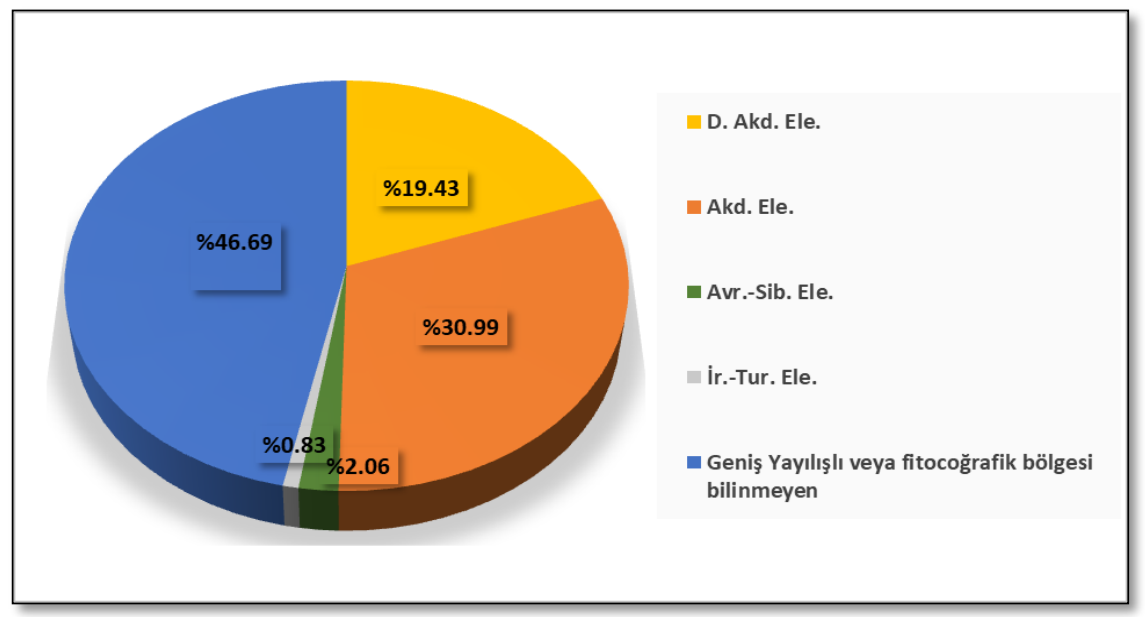

Şekil 5. Araştırma alanındaki bitki taksonlarının fitocoğrafik bölgelere göre dağılımı

Tablo 2. Çalışma alanımız ve yakın bölgelerde yapılmıș diğer çalışmaların endemizm oranları

\begin{tabular}{|c|c|c|c|c|c|}
\hline Araştırma alanı & $\begin{array}{c}\text { İztuzu Plajı ve } \\
\text { Çevresinin } \\
\text { Florası }\end{array}$ & $\begin{array}{c}\text { Köyceğiz- } \\
\text { Dalyan Özel } \\
\text { Koruma } \\
\text { Alanının Florası } \\
\text { [12] }\end{array}$ & $\begin{array}{c}\text { Aspat (Strobilos) } \\
\text { Kalesi ve } \\
\text { Çevresinin } \\
\text { Floristik } \\
\text { Özellikleri [13] }\end{array}$ & $\begin{array}{c}\text { Urban Flora of } \\
\text { Muğla (Muğla, } \\
\text { Turkey) [14] }\end{array}$ & $\begin{array}{c}\text { Floristic } \\
\text { Structure of } \\
\text { Historical } \\
\text { Labranda Ruins } \\
\text { and its } \\
\text { Surroundings } \\
\text { (Milas, } \\
\text { Muğla/Turkey) } \\
\text { [15] }\end{array}$ \\
\hline Endemizm & $\begin{array}{c}7 \\
(\% 2.89)\end{array}$ & $\begin{array}{c}78 \\
(\% 8.78)\end{array}$ & $\begin{array}{c}13 \\
(\% 3.85)\end{array}$ & $\begin{array}{c}30 \\
(\% 5.20)\end{array}$ & $\begin{array}{c}18 \\
(\% 5.37)\end{array}$ \\
\hline
\end{tabular}


Tablo 3. Çalışma alanımızdaki endemik bitki ve tehlike kategorileri

\begin{tabular}{|l|c|}
\hline \multicolumn{1}{|c|}{ Endemik Bitkiler } & Tehlike Kategorileri \\
\hline $\begin{array}{l}\text { Arenaria pamphylica subsp. pamphylica } \\
\text { var. pamphylica }\end{array}$ & VU \\
\hline Cyclamen alpinum & LC \\
\hline Alkanna mughlae & EN \\
\hline Aristolochia guichardii & VU \\
\hline Quercus aucheri & LC \\
\hline Galium brevifolium subsp. brevifolium & LC \\
\hline Fritillaria sibthorpiana subsp. enginiana & EN \\
\hline
\end{tabular}

Araștırma alanımız ve Muğla'da yapılan bazı floristik çalışmalar en fazla takson içeren familyalar bazında Tablo 4'de karşılaştırılmıştır. Tabloya bakıldığında; içerdikleri takson sayısı bakımından familyalar bütün alanlarda karşılaştırıldığında çalışma alanımız, Köyceğiz-Dalyan Özel Koruma Alanı ve Aspat Kalesi ve çevresinde Asteraceae, Fabaceae ve Poaceae familyalarının baskın durumda olduğu görülmektedir. Muğla'nın urban florasında Asteraceae ve Fabaceae familyaları baskın durumda iken, Poaceae familyasının oranı daha düşüktür. Aynı alanda Poaceae, Brassicaceae ve Lamiaceae familyalarının hemen hemen eşit oranlarda bulunduğu görünmektedir. $\mathrm{Bu}$ durumun; çalışma alanımızın, Köyceğiz-Dalyan Özel Koruma Alanının ve Aspat Kalesi'nin deniz seviyesinde olması, Muğla şehir merkezinin ise 600 m yükseltide olması ve şehir merkezinde yerleșim birimlerinin bulunmasından kaynaklandığı düşünülmektedir.

Araştırma alanımız ve Muğla'da yapılan bazı floristik çalışmalar en fazla takson içeren cinsler bazında karşılaştırıldığında baskın durumda olan Trifolium L. cinsidir. Trifolium cinsi Akdeniz bölgesinde lokalize olan bir cinstir. Genellikle ekolojik toleransı fazla ve geniş yayılışa sahip tür ve türaltı taksonları içerir. Köyceğiz-Dalyan Koruma alanının genişliği, farklı habitat tiplerini içermesi ve sahip olduğu takson sayısının fazla olmasına bağlı olarak, cins sayısının da diğer alanlardan daha fazla oranda bulunması dikkat çekmektedir. Çalışma alanımız ve diğer alanlarda Trifolium dışında bulunan cinsler birbirine yakın oranlarda bulunmaktadır (Tablo 5).

Tablo 4. En fazla takson içeren ilk 10 familyanın yakın bölgelerde yapılmış diğer çalışmalarla karşılaştırılması

\begin{tabular}{|c|c|c|c|c|c|}
\hline Familyalar & $\begin{array}{l}\text { İztuzu Plajı ve } \\
\text { Çevresinin } \\
\text { Florası }\end{array}$ & $\begin{array}{l}\text { Köyceğiz- } \\
\text { Dalyan Özel } \\
\text { Koruma } \\
\text { Alanının } \\
\text { Florası [12] }\end{array}$ & $\begin{array}{c}\text { Aspat } \\
\text { (Strobilos) } \\
\text { Kalesi ve } \\
\text { Çevresinin } \\
\text { Floristik } \\
\text { Özellikleri } \\
\text { [13] }\end{array}$ & $\begin{array}{c}\text { Urban Flora of } \\
\text { Muğla (Muğla, } \\
\text { Turkey) [14] }\end{array}$ & $\begin{array}{c}\text { Floristic } \\
\text { Structure of } \\
\text { Historical } \\
\text { Labranda } \\
\text { Ruins and its } \\
\text { Surroundings } \\
\text { (Milas, } \\
\text { Muğla/Turkey) } \\
\text { [15] }\end{array}$ \\
\hline Asteraceae & $28(\% 11.57)$ & $91(\% 9.84)$ & $57(\% 16.91)$ & $54(\% 9.37)$ & $41(\% 12.23)$ \\
\hline Poaceae & 27 (\%11.15) & $93(\% 10.06)$ & $20(\% 5.93)$ & $33(\% 5.72)$ & $23(\% 6.86)$ \\
\hline Fabaceae & $25(\% 10.33)$ & $92(\% 9.95)$ & $44(\% 13.05)$ & $64(\% 11.11)$ & $40(\% 11.94)$ \\
\hline Brassicaceae & $13(\% 5.37)$ & $38(\% 4.11)$ & $13(\% 3.85)$ & $36(\% 6.25)$ & $15(\% 4.47)$ \\
\hline Lamiaceae & $12(\% 4.95)$ & $43(\% 4.65)$ & $15(\% 4.45)$ & $33(\% 5.72)$ & $14(\% 4.20)$ \\
\hline Apiaceae & $12(\% 4.95)$ & $44(\% 4.76)$ & $11(\% 3.35)$ & $19(\% 3.29)$ & $11(\% 3.28)$ \\
\hline Caryophyllaceae & $11(\% 4.54)$ & $38(\% 4.11)$ & $13(\% 3.85)$ & $28(\% 4.86)$ & $20(\% 5.97)$ \\
\hline Plantaginaceae & $8(\% 3.30)$ & $9(\% 0.97)$ & $5(\% 1.48)$ & $5(\% 0.86)$ & $13(\% 3.90)$ \\
\hline Euphorbiaceae & $7(\% 2.89)$ & $19(\% 2.05)$ & $3(\% 0.89)$ & $5(\% 0.86)$ & $5(\% 1.50)$ \\
\hline Ranunculaceae & $7(\% 2.89)$ & $19(\% 2.05)$ & $4(\% 1.18)$ & $18(\% 3.11)$ & $4(\% 1.20)$ \\
\hline
\end{tabular}


Tablo 5. En fazla takson içeren ilk 10 cinsin yakın bölgelerde yapılmıș diğer çalıșmalarla karșılaștırılması

\begin{tabular}{|c|c|c|c|c|c|}
\hline Cinsler & $\begin{array}{l}\text { İztuzu Plajı ve } \\
\text { Çevresinin } \\
\text { Florası }\end{array}$ & $\begin{array}{c}\text { Köyceğiz- } \\
\text { Dalyan Özel } \\
\text { Koruma } \\
\text { Alanının } \\
\text { Florası [12] }\end{array}$ & $\begin{array}{c}\text { Aspat } \\
\text { (Strobilos) } \\
\text { Kalesi ve } \\
\text { Çevresinin } \\
\text { Floristik } \\
\text { Özellikleri [13] }\end{array}$ & $\begin{array}{c}\text { Urban Flora of } \\
\text { Muğla (Muğla, } \\
\text { Turkey) [14] }\end{array}$ & $\begin{array}{c}\text { Floristic } \\
\text { Structure of } \\
\text { Historical } \\
\text { Labranda } \\
\text { Ruins and its } \\
\text { Surroundings } \\
\text { (Milas, } \\
\text { Muğla/Turkey) } \\
\text { [15] } \\
\end{array}$ \\
\hline Trifolium & 9 & 16 & 15 & 12 & 14 \\
\hline Euphorbia & 6 & 16 & 2 & 4 & 4 \\
\hline Ranunculus & 4 & 10 & 2 & 11 & 4 \\
\hline Bromus & 4 & 10 & 3 & 4 & 3 \\
\hline Plantago & 4 & 9 & 5 & 5 & 5 \\
\hline Galium & 3 & 10 & 3 & 7 & 4 \\
\hline Anthemis & 3 & 6 & 3 & 5 & 2 \\
\hline Medicago & 3 & 14 & 7 & 6 & 6 \\
\hline Allium & 3 & 16 & 4 & 6 & 3 \\
\hline Veronica & 3 & 7 & 1 & 5 & 6 \\
\hline
\end{tabular}

780.000 km²'lik yüzölçümüne sahip olan Türkiye'de 12000'e yakın bitki taksonunun varlığı göz önüne alındığında, 4,5 km²’lik küçük bir alan olan İztuzu sahili ve çevresinde 242 bitki taksonunun tespit edilmiş olması bu alanın bitki biyoçeşitliliği bakımından ne kadar zengin olduğunun bir göstergesidir.

Alanda bulunan Pancratium maritimum L. (Kum zambağı) kıyı kumullarında yetişen soğanlı bir bitki türüdür. Ancak doğal yașam alanı olan sahillerin plaj olarak kullanılması ve koruma önlemlerinin alınmaması sebebiyle, türün populasyonunun günümüzde ciddi anlamda tehdit altında olduğu görülmektedir. Yaptığımız bu çalışma esnasında kum zambaklarının populasyonu üzerine de gözlemler yapılmış olup, gösterişli çiçeklere sahip olan bu bitkimizin İztuzu sahili içerisinde populasyonunun arttırılmasına yönelik koruma biyolojisi çalışmalarının yapılması büyük bir önem arz etmektedir.

Sonuç olarak bu çalışmanın Türkiye florasına katkıda bulunacağı, ileride bu bölgede yapılacak sosyolojik ve diğer yakın bölgelerde yapılacak floristik çalıșmalara ıșı tutacağı, aynı zamanda kum zambağı bitkisini korumaya yönelik farkındalık oluşturacağı kanaatindeyiz.

\section{Kaynakça}

[1] Seçmen, Ö. 2008. Türkiye Florası (Dersv Notları). Ege Üniversitesi, Fen Fak. Baskı İşleri, Bornova, İzmir, 5s.

[2] Güner, A., Aslan, S., Ekim, T., Vural, M., Babaç, M.T. (edlr.) 2012. Türkiye Bitkileri Listesi
(Damarlı Bitkiler). Nezahat Gökyiğit Botanik Bahçesi ve Flora Araştırmaları Derneği Yayını, İstanbul.

[3] Eken, G., Bozdoğan, M., İsfendiyaroğlu, S., Kılıç, DT., Lise, Y.. 2006. Türkiye'nin Önemli Doğa Alanları. Doğa Derneği, İstanbul: Mas Matbaacilık.

[4] Davis, P.H. 1965. Flora of Turkey and The East Aegean Islands. Edinburgh University Press, Vol. 1., Edinburgh.

[5] MTA 1997. 1 / 100000 Ölçekli Fethiye L-7 Paftasi.

[6] Anonim, 1998. Muğla İli Arazi Varlığı, T.C. Başbakanlık Köy Hizmetleri Genel Müdürlüğü Yayınları, Ankara.

[7] Emberger, L. (1952) Sur le Quotient pluviothermique, C.R. Acad. Sci., 234, 25082510.

[8] Akman, Y. (1990) İklim ve Biyoiklim, Palme Yayın Dağıtım, Ankara, 320s.

[9] Davis, P. H., Mill, R. R., Tan, K. (eds). 1988. Flora of Turkey and East aegean Islands. Vol.10, Supplement, Edinburg.

[10] Güner, A., Özhatay, N., Ekim, T., Başer, K.H.C. (eds.) 2000. Flora of Turkey and the East Aegean Islands, (Suppl. 2). Vol. 11, Edinburgh University Press. Edinburgh, UK. 221p.

[11] Tekşen, M., Aytaç, Z. 2011. The revision of the genus Fritillaria L. (Liliaceae) in the Mediterranean region (Turkey). Turk J Bot, 35: 447-478. 
[12]Güner, A., Vural, M., Duman, H., Dönmez H., Şağban, H. 1996. The flora of the KöyceğizDalyan Specially Protected Area (MuğlaTurkey). Turkish Journal of Botany, 20: 329372.

[13]Çınar, H. 2010. Aspat (Strabilos) Kalesi ve Çevresinin Floristik Özellikleri. Yüksek Lisans Tezi, Muğla Sıtkı Koçman Üniversitesi, Muğla.

[14] Kaya, E., Varol Ö., Aytepe, H. 2008. Urban Flora of Muğla (Muğla, Turkey). Flora Mediterranean, 18: 127-148.

[15] Güler, B., Varol, Ö. 2012. Floristic structure of historical Labranda ruins and its surroundings (Milas, Muğla/Turkey). Biological Diversity and Conservation, 5(3), 54-68.
[16] IUCN, 2003. Red List Categories: Version 3.1. Prepared by the IUCN Species Survival Commission. Gland, Switzerland.

[17] Ekim, T., Koyuncu. M., Vural, M., Duman, H., Aytaç, Z., Adıgüzel, N. 2000. Türkiye Bitkileri Kırmızı Kitabı. Yayın No: 18, Ankara, 246s.

\section{Simgeler ve Kısaltmalar:}

Akd. Ele.: Akdeniz Elementi, D. Akd. Ele.: Doğu Akdeniz elementi, İr.-Tur. Ele.: İran-Turan Elementi, Avr..-Sib. Ele.: Avrupa-Sibirya elementi, EN: Tehlikede, End.: Endemik, Lok.: Lokalite, LC: Düşük Riskli, m: Metre, Ö.V: Ömer VAROL, subsp.: Alttür, var.: Varyete, VU: Zarar görebilir 